\section{P155 IMMO-LDRT01 TRIAL: IMMUNOMODULATORY EFFECTS OF LOW DOSE RADIATION THERAPY OF CHRONIC DEGENERATIVE AND INFLAMMATORY DISEASES}

A-J Donaubauer*, T Gryc, I Becker, OJ Ott, R Fietkau, US Gaipl, B Frey. Radiation Oncology, University Hospital Erlangen, Erlangen, Germany

\subsection{6/annrheumdis-2018-EWRR2019.137}

Career situation of first and presenting author Student for a master or a $\mathrm{PhD}$.

Introduction The treatment of chronic inflammatory and/or degenerative diseases by low dose radiation therapy (LDRT) is a successful alternative treatment option, especially for patients who were refractory for treatment with drugs. Generally, LDRT aims to reduce pain of patients and to increase their mobility. Although LDRT has successfully been applied since the late 19th century, the underlying immunological mechanisms are only fragmentarily analyzed.

Objectives Therefore we have aimed to analyze the impact of locally delivered LDRT on systemic immune changes.

Methods For this the IMMO-LDRT01 study (NCT02653079) was initiated in 2016. The study will include 150 patients suffering from chronic inflammatory and/or degenerative diseases, such as arthritis, arthrosis or benign calcaneodynia. The patients are treated with six local irradiations (single dose per fraction of $0.5 \mathrm{~Gy}$ ) in three weeks. If necessary, the serial irradiation can be repeated after 8-12 weeks, in order to achieve a further reduction of the pain. Blood samples are taken before and after every serial irradiation, as well as during follow-up appointments of the patients. For detailed immunophenotyping of whole blood, we established a multicolor flow cytometry assay, which allows the monitoring of seven main immune cell types, 26 immune cell subtypes, and the activation status of the immune cells.

Results To date 80 patients have been included in the study. First evaluations showed a significant reduction of B cells and cytotoxic $T$ cells in the peripheral blood of patients. In particular, LDRT impacted on activation markers of immune cells. One has to stress that the immunological effects of LDRT were dependent on the particular type of disease of the patients. Currently, the data of the immunophenotyping are correlated to pain perception and quality of life of the patients.

Conclusions These analyses will be helpful to optimize LDRT. A better patient stratification based on pain- and immunerelated biomarkers is envisaged for the future, alongside with initiation of deliberated randomized clinical trials.

Acknowledgements This study has been in part funded by the Bundesministerium für Bildung und Forschung (GREWIS and GREWIS-alpha, 02NUK017G and 02NUK050E).

Disclosure of Interest None declared.

\section{P156/008 SYSTEMIC INFLAMMATION ORIGINATING FROM THE SKIN OR THE INTESTINE COMBINED WITH BIOMECHANICAL STRESS HAS DIFFERENT EFFECTS ON THE JOINTS IN AN IN VIVO MOUSE MODEL}

${ }^{1} \mathrm{GR}$ Gulino*, 1,2M Van Mechelen, 1,2R Lories. 'Skeletal Biology and Engineering Research Center, KU Leuven; ${ }^{2}$ Division of Rheumatology, UZ Leuven, Leuven, Belgium

10.1136/annrheumdis-2018-EWRR2019.138

Career situation of first and presenting author Post-doctoral fellow.
Introduction Psoriasis (PsO) and inflammatory bowel disease (IBD) share a wide range of comorbidities, including psoriatic arthritis (PsA). Entheses, the attachment sites of tendons and ligaments into the bones, spread the mechanical forces generated by movement onto the bone and are considered as a primary disease localization in PsA. Increasing evidence supports the hypothesis that biomechanical stress, together with inflammatory triggers from distant sites, such as the skin or the intestine, can contribute to the onset of PsA by inducing microdamage in the entheses.

Objectives In this study, we aimed to investigate the role of biomechanical stress together with cutaneous or intestinal inflammation in initiating joint disease.

Methods Eight-week-old male C57/B16 mice were treated with imiquimod cream (IMQ) on a shaved area of the back skin or with dextran sodium sulphate (DSS) dissolved in the drinking water to induce PsO-like skin or IBD-like gut inflammation. Control mice were left untreated. Afterwards, half of the mice were subject to a forced treadmill running protocol to increase biomechanical stress. Control mice with or without IMQ or DSS treatment did not run. Severity of cutaneous or intestinal inflammation was assessed clinically and by histology; knees and paws were analyzed by microCT, histology and immunohistochemistry.

Results Local induction of cutaneous or intestinal inflammation led to a systemic response, as detected by splenomegaly, trabecular subchondral bone loss and bone marrow hypercellularity.

On a background of PsO- but not of IBD-like inflammation, discrete signs of synovitis determined by the presence of CD45 + cells and overexpression of IL-17 were detected, with no significant impact of the running protocol. The CD45+ cells in the IMQ non-running condition were identified as F4/80+cells, in contrast to the running condition where the CD45+ cells could not be further identified, showing negative results for F4/ 80, CD3, MPO and MCSF-R stainings.

Furthermore, forced exercise and PsO-like inflammation both induced overexpression of IL-17 at the entheses and acted synergistically when combined.

Conclusions Local induction of PsO- or IBD-like inflammation triggers a systemic response with inflammation-associated bone loss and discrete signs of joint disease. PsO-like inflammation in combination with biomechanical stress increased the degree of synovitis and enthesitis, showing that systemic inflammation combined with biomechanical stress may contribute to disease manifestations in PsA.

Disclosure of Interest G. Gulino: None declared, M. Van Mechelen: None declared, R. Lories Consultant for: Abbvie, Celgene, Eli Lilly, Janssen, Novartis, Pfizer, UCB, Speakers bureau: Abbvie, Celgene, Eli-Lilly, Janssen, Novartis, Pfizer, UCB.

\section{P157 RADIORESISTANT AND RADIOSENSITIVE CELLS CONTRIBUTE TO IL-18BP PRODUCTION IN A MODEL OF MACROPHAGE ACTIVATION SYNDROME}

${ }^{1} \mathrm{M}$ Harel ${ }^{*},{ }^{1} \mathrm{E}$ Rodriguez, ${ }^{2} \mathrm{C}$ Girard-Guyonvarc' $\mathrm{h},{ }^{1} \mathrm{G}$ Palmer, ${ }^{1} \mathrm{C}$ Gabay. ${ }^{1}$ Pathology and Immunology, University of Geneva School of Medicine; ${ }^{2}$ Department of Internal Medicine Specialties, Division of Rheumatology, Geneva, Switzerland

10.1136/annrheumdis-2018-EWRR2019.139

Career situation of first and presenting author Student for a master or a $\mathrm{PhD}$. 